University of the Pacific

Scholarly Commons

8-1-2002

\title{
Diagnosing stuttering in the school environment
}

Michael Susca

University of the Pacific, msusca@pacific.edu

Follow this and additional works at: https://scholarlycommons.pacific.edu/shs-all

Part of the Medicine and Health Sciences Commons

\section{Recommended Citation}

Susca, Michael, "Diagnosing stuttering in the school environment" (2002). All Faculty Scholarship. 43. https://scholarlycommons.pacific.edu/shs-all/43

This Article is brought to you for free and open access by the School of Health Sciences at Scholarly Commons. It has been accepted for inclusion in All Faculty Scholarship by an authorized administrator of Scholarly Commons. For more information, please contact mgibney@pacific.edu. 


\title{
Diagnosing Stuttering in the School Environment
}

\author{
Michael Susca, Ph.D., CCC-SLP1
}

\section{ABSTRACT}

School speech-language pathologists work in settings that present multiple challenges to diagnosing stuttering in students. Perceiving stuttering as a communication impairment allows integration of various issues with criteria from the Individuals with Disabilities Education Act 1997 into an effective diagnostic plan. Assessment from multiple perspectives reveals motor, linguistic, social, affective, and cognitive issues related to communication and educational competencies. Common sense and pragmatic communication concerns related to children who stutter are described. Clinical questions, observations, issues, and tasks are explored as parts of the assessment process. The multidimensional assessment of student-centered issues helps the clinician determine eligibility qualifications, develop individual education plans, and meet full-inclusion educational requirements.

KEYWORDS: Diagnosis, assessments, stuttering, service eligibility, school-aged children

Learning Outcomes: As a result of this activity, the participant will be able to (1) understand how diagnosing multiple factors contributing to stuttering can be integrated with IDEA '97 principles, (2) use multiple perspectives to determine eligibility for speech services in the schools, and (3) integrate various diagnostic strategies to design effective individualized education plans that allow full-inclusion educational experiences.

Diagnosing stuttering in school-aged children can be a challenging task for the school-based speech-language pathologist, for many reasons. One key challenge is the fact that stuttering is highly variable: it changes across time and across situations. Due to this variability, stuttering behaviors may be reported by parents or teachers but not observed by clinicians during the brief time allotted for assessment. School systems often require a quick diagnosis in an environment where the clinician has responsibilities to large caseloads

Facing the Challenge of Treating Stuttering in the Schools. Part I: Selecting Goals and Strategies for Success; Editors in Chief, Nancy Helm-Estabrooks, Sc.D., and Nan Bernstein Ratner, Ed.D.; Guest Editor, J. Scott Yaruss, Ph.D. Seminars in Speech and Language, volume 23, number 3, 2002. Address for correspondence and reprint requests: Michael Susca, Ph.D, Department of Speech Language Pathology, University of the Pacific, 1050 Brookside Road, Stockton, CA 95211. E-mail: msuscal@uop.edu. ${ }^{1}$ University of the Pacific, Stockton, California. Copyright (C) 2002 by Thieme Medical Publishers, Inc., 333 Seventh Avenue, New York, NY 10001, USA. Tel: +1(212) 584-4662. 0734-0478,p;2002,23;03, 165,172 ,ftx,en;ss1119x. 
of children with other communication needs. Another challenge school clinicians frequently face is a lack of direct parental access and involvement in the diagnostic process. Finally, many school clinicians are simply uncomfortable working with students who stutter. ${ }^{1}$ These challenges, coupled with school system requirements for using standardized assessment to determine service eligibility and the need to tie diagnostic recommendations to educational criteria, put the school clinician at a distinct disadvantage for diagnosing stuttering in the school-aged population.

This disadvantage can be partially overcome by relinquishing the narrow perspective of stuttering as a "speech problem" and engaging the broader perspective of stuttering as a communication problem. The observable characteristics of stuttering are only one component contributing to the communication problems experienced by students who stutter. Other contributing components such as temperament, environment, language, physiology, emotions, and cognition have been identified as contributors and have led to a larger conceptualization of the problem. ${ }^{2-4}$ Beyond these components are multiple levels of reactions to stuttering: the child's reactions to his own stuttering, others' (peers, parents, teachers) reactions to his stuttering, and the child's reactions to those reacting to the stuttering. Stuttering has come to be understood from a multifactorial, nonlinear, and dynamic framework. ${ }^{5}$ The current consensus is that stuttering is a multidimensional disorder with multiple interactive components and, as such, is really best viewed as a communication problem rather than a speech problem.

According to the Individuals with Disabilities Education Act of 1997 (IDEA '97), certain criteria regarding eligibility qualifications, evaluation procedures, evaluation data, and particular procedures are required to determine eligibility for school-based speech and language services. The law allows children who have communication impairments to be placed in a category of disability that qualifies them for services. The school-based speech-language pathologist should know that the definition of disability that satisfies eligibility requirements includes "... a communication disorder, such as stuttering ... that adversely affects a child's educational performance." 6 Although the adverse impact is not defined by the law, educational performance is determined individually and "should include nonacademic as well as academic areas" (Department of Education, 1990). Thus, assessment must be directed to a child's functional ability to participate in curricular and social activities.

IDEA '97 clearly specifies that assessment procedures for determining eligibility are specifically not restricted to standardized measures. In fact, a multidisciplinary team and multilevel approach is supported, which includes interviews with a variety of information sources: parents, teachers, the child, and other significant school personnel. Through these interviews, relevant information may be gathered regarding functional communication abilities in multiple conditions and environments. A determination can be made of the presence, extent, and relative importance of a fluency disorder. This information can be directly used in the development of treatment recommendations. Each interview may focus on any one or the combination of motor, linguistic, affective, cognitive, social, or behavioral issues associated with the communication problem. Questions may be direct or indirect and will be influenced by the experience or comfort level of the clinician with any individual interviewed. Examples of questions pertinent to the child's functioning within the school environment include the following.

- How does the child deal with feelings of anticipation during oral readings in class?

- Are there times when it is difficult to talk? For example, when answering questions aloud in class, telling a joke's punch line, or requesting information or items from classmates?

- Does the child like talking? Does it matter to the child?

- Do peers make fun, tease, or ridicule the child regarding his speech?

- Is there difficulty asking questions or directions from other teachers, counselors, or office personnel? What is the difficulty? 
- Does the student do anything special to make talking easier?

Responses to questions like these will help reveal the student's general communicative functioning. Additional questioning will reveal academic history and experiences, family history, temperament, and any idiosyncratic concerns that may affect a decision regarding the presence or absence of a fluency problem. Also, the speech-language pathologist might want to note various medical issues (e.g., attention deficit disorder, neuromuscular deficits) that may also contribute to or be a part of fluency and communication problems. Such background information obtained by the clinician directly influences the direction of any recommended treatment.

Obviously, the very fact of the referral to the school speech-language pathologist may be a basis for determining the presence of the problem for a school-aged child. Because the student is school-aged, there is an implied likelihood that any existing stuttering will continue without intervention. Thus, the next question becomes, "Is this the right time or place for treatment?" When interviewing the student important areas of assessment will include the child's degree of concern, motivation to do something about it, and proposed conditions of treatment (e.g., pull-out, during school breaks, at lunch, in natural activities, after school) with respect to the fluency problem. Many children who stutter simply do not want to be identified and treated differently from their nonstuttering peers. Lack of motivation or concern about the problem may be mediated by the student's feelings of fitting in the school social environment. That is, concerns about peers' perception of the student needing "special" services may undermine the effectiveness of any proposed intervention. Although diagnosed with a stuttering problem affecting communication and ability to have a fully included educational experience, some individuals may not be candidates for traditional intervention strategies. Instead, the clinician may need to engage in creative intervention strategies that are consultative, indirect, or generally supportive. That is, an evaluation may reveal a therapy need but mitigating circumstances, which preclude direct intervention, might require clinical adjustment or modification in treatment planning and provision.

When assessing the student who is identified as a child who stutters, the speech-language pathologist needs to include evaluation of functioning in other communication-related skills as well. Specifically, this includes the student's functioning in the areas of articulation, language, sociability, and cognition. Speech motor control difficulties have been identified in as many as $68 \%$ of young children who stutter. ${ }^{7}$ Specific articulation difficulties are so common in children who stutter that school-based speech-language pathologists can anticipate articulation problems in about one fourth to one third of their caseload of children who stutter. ${ }^{8}$ Sometimes the challenge is determining if the oral motor and articulation problems are coexisting, contributing to, or the result of fluency disruptions. To a lesser extent, disturbances in language and voice also have been identified as coexisting or contributing to disruptions in fluency; these factors should also be evaluated.

The school speech-language pathologist may also want to explore how the stuttering is related to communication in social contexts. Does the student withdraw from or not participate in verbal exchanges and games with peers? In other words, does the student experience periods of social shyness or withdrawal because of communication fluency problems? The student's thoughts about and reactions to his speech need also be determined. What is the student's level of awareness of the fluency problem and what are his reactions to disruptions in fluency? Does avoidance, substitution, or circumlocution of sounds, words, or situations occur because of speech disfluencies? What does the student think of his speech as it relates to his social and academic functioning? What is the student telling himself about his abilities/limitations vis-à-vis his speech? Questions such as these may reveal the presence of stuttering disorder without the presence of observable symptoms.

In the search for observable signs of stuttering, many school-based speech-language 
pathologists feel compelled to conduct a "standardized" diagnostic assessment. Such an assessment alone is insufficient and in direct violation of the intent of IDEA '97. Still, the many demands placed on the school clinician often result in the use of a quick standardized assessment such as The Stuttering Severity Instrument for Children and Adults-Third Edition (SSI-3). ${ }^{9}$ SSI-3 scores may fail, however, to accurately reflect a considerable range of observations underlying stuttering behaviors. ${ }^{10} \mathrm{~A}$ frequent limitation of single instrument assessments of observable stuttering is the failure to account for the linguistic and situational variability so commonly associated with stuttering. That is, a major shortcoming of many instruments measuring observable stuttering is that only surface features of the communication problem are assessed, encouraging a narrow perspective of the disorder. Although not required, having numerical counts of stuttering behaviors often helps the school speech-language pathologist "make her case" for including a child in therapy. One might wonder, however, if making a case on the basis of observable characteristics is for the benefit for the clinician, the child, or the bureaucracy.

When collecting speech samples from a student, a 300- to 500-word sample in two or more conditions is a minimum. Speech samples will vary with the student's age, motivation, temperament, and availability but should be gathered in various environments (i.e., classroom, hallway, resource room, lunch room) and in multiple situations (i.e., oral reading, group activity, using a telephone, peer conversation during lunch periods). Sometimes a student is fluent in the presence of the school clinician regardless of environment, situation, or task load. When speech fluency is highly variable, the speech-language pathologist may need to adjust linguistic, cognitive, or emotional loads in the sampling task as necessary. In such cases, the speech-language pathologist may want to provide direct communication stresses (e.g., appearing disinterested in student comments, confrontational challenges of student statements, or interruptions of student expressions.) Such communicative stresses, when appropriately applied, may reveal stutter- ing that is otherwise unobserved. Clearly, a one-on-one sample taken with the speechlanguage pathologist in an isolated room on a pull-out basis is not representative of the student's fluency or level of communicative functioning. In many cases, the speech-language pathologist can ask the student if the samples collected are representative of the student's general level of speech fluency and/or stuttering.

Once samples are collected, measurement may include the total number of disfluent moments as well as stuttering types and frequencies. Either syllables or words may be counted in a sample, as there are no hard data suggesting one method is better than another. It is important, nonetheless, to be clear and consistent regarding the method used. Again, speech-language pathologists are directed by IDEA '97 to not rely solely on a single test score or set of scores. Guidelines are available based on observable characteristics, but it must be kept in mind that they do not show the whole picture. A student may be naturally very disfluent but not clearly stuttering. Some characteristics a school clinician might observe to help differentiate stuttering from normal disfluencies are the following.

- The number of total disfluencies is greater than $10 \%$.

- Sixty-six percent to $81 \%$ of total disfluencies are stuttering-like disfluencies. ${ }^{12}$

- Sound prolongations exceed 1 second in duration.

- Thirty percent or more stuttered events consist of sound prolongations.

- There are irregular phonatory characteristics (vocal tension, vocal fry, voice stoppages, abnormal inflections).

- There is an observable (seen or heard) struggle on greater than $3 \%$ of utterances.

Once again, the author cautions that many students who stutter will not show these symptoms as they have achieved a level of sophistication that allows them to "hide" the observable stutter.

As many clinicians have noted, the majority of stuttering is unobservable. Assessment needs to be directed to the evaluation of communication issues related to stuttering that are 
not easily observed or measured. Commonly, at some point during or after the development of stuttering, a person who stutters begins to perceive the ability to communicate differently from a person who does not stutter. This perception may permeate the individual's selfconcept and result in altered feelings, thoughts, and/or behaviors with respect to communication. Thus, many unobservable components of the disorder may play greater roles in the development and expansion of stuttering than the observable stuttering behaviors alone. For example, fears and anxieties about communication in general or with particular sounds, words, persons, or situations may develop and lead to the commonly observed secondary characteristics of avoidance, substitutions, and circumlocutions. Other students learn to use various fluency enhancing conditions to modify or cover up the stuttering. For example, some may seem to truncate verbal responses to questions, speak with altered rhythms or accents, use the schwa vowel or throat clearing behaviors to initiate utterances, or "act out" behaviorally or verbally to postpone desired responses. In the last example, such behaviors may result in the label of "class clown." The student who can internally anticipate and modify stuttering before it is externally expressed often has odd language patterns or peculiar language pragmatics. In the process of the struggle for fluency, attitudes about self and communication become modified, thoughts occur about being different, and competence in social and academic contexts is negatively affected.

Several published protocols are available to the school clinician to observe and measure these subtle communication issues. Examples include Burks Behavior Rating Scale, ${ }^{12}$ The Communicative Attitude Test-Revised, ${ }^{13} \mathrm{Be}-$ havioral Scale Questionnaire, ${ }^{14}$ Speech Situation Checklist, ${ }^{15}$ The A-19 Scale, ${ }^{16}$ and the Fear Survey Schedule. ${ }^{17}$ These protocols direct the speech-language pathologist's attention to the student's possible negative emotional feelings, situations associated with speech disruptions, attitudes about talking, fears associated with talking, self-expectations, or general frustration tolerances and sensitivity levels that may be part of the communication problem. Another area of assessment includes determining the student's general adaptability or ability to make changes. Assessment of this area may point to the likelihood of therapeutic effectiveness. Gauging the student's responses to time pressure and tension experienced physically or emotionally may affect how he will respond to therapy. Reactions to novel experiences, new ways of doing behaviors or dealing with environmental changes may also have prognostic value. Finally, examining the student's beliefs and thoughts about stuttering and responses to stuttering will directly affect motivation to modify his communicative experiences.

The protocols listed are only offered as a "last resort" for those speech-language pathologists uncomfortable or unsure of what to assess in the affective or cognitive areas of functioning. There are also comprehensive kits available that contain checklists that address both the attitudes of people who stutter and the attitudes of significant others related to them. Two examples of published kits are the Personalized Fluency Control Therapy ${ }^{18}$ by E. Cooper and the Behavioral Cognitive Stuttering Therapy ${ }^{19}$ by S.A. Goldberg.

Through the process of assessment, the speech-language pathologist will identify the specific fluency and communicative concern(s) and who has the concern(s). When a stuttering problem is identified, its relationship to academic, social, and life issues will become clear. Academic issues may include ability to participate in group projects, give oral reports, or simply ask or respond to questions in class. A student may not reveal his stutter as observable speech events but does reveal the communication disorder through minimal participation in educational matters. Such a student is a good candidate for therapy designed to aid the student towards full inclusion in the educational process. In contrast, another student who openly stutters, is well adjusted to the fluency disruptions, and fully participates in academic and nonacademic activities may not be an immediate candidate for therapy.

Social issues affected by stuttering may include participation in the dynamics of group 
lunch conversations, games at recess, and discussion of current events (in or out of class). A student who cannot respond with efficient timing of utterances may be precluded from engaging in verbal repartee, sport commentaries, or quick expressions of opinion about a popular movie. A student with these experiences may become socially isolated, demonstrate lower self-esteem, or express frustration, shame, and/or guilt regarding relationships with peers. These experiences may also result in negative self-talk and feelings of helplessness, negatively affecting academic, nonacademic, and extracurricular activities. Because the socially withdrawn student is not verbally active, stuttering is not viewed as problematic, but the communication impairment is significant in that it precludes involvement in the total school experience.

The diagnosis of stuttering will often reveal interacting components associated with the stuttering and communication in the educational environment. The integrative evaluation process will lead to a determination of eligibility in accordance with the law. IDEA '97 provides the means for school-based speechlanguage pathologists to meet the varied needs of students who stutter.

It has already been stated that stuttering is a dynamic, multidimensional disorder. Components contributing to or maintaining the problem will differ from student to student as well as within a single student across his educational development. Therefore, the school speech-language pathologist will need to regularly assess the constellation of components contributing to a particular student's communication problem and any resultant adverse educational impact. For example, in one case the assessment may point to a need for educating classmates and teachers about stuttering and communication pragmatics during daily classroom activities. In another case, the assessment may point to the need to help the student deal with teasing, bullying, or other negative reactions of peers. In still another case, the focus may be directed to developing stuttering modification techniques to manage verbal communication in particular educational contexts. The point remains: a differential diagnosis of stut- tering that is interwoven in the fabric of multiple educational experiences will provide the necessary information to both meet eligibility requirements and formulate an effective individualized education plan.

\section{ACKNOWLEDGMENTS}

The author wishes to express gratitude to many individuals who have provided background information to develop this article. Special thanks go to the many school speech therapists who expressed their experiences about provision of services in the school environment. Thanks also are given to Lisa Trautman regarding background issues related to IDEA and Susan Blanchard regarding practical administration matters of school-wide services. Finally, the author thanks Scott Yaruss for guiding the direction of this article.

\section{REFERENCES}

1. Brisk DJ, Healey EC, Hux KA. Clinicians' training and confidence associated with treating school-age children who stutter: a national survey. Lang Speech Hear Serv Schools 1997;28: 164-176

2. Meyers F, Wall M. Toward an integrated approach to early childhood stuttering. J Fluency Disord 1982;7:47-54

3. Zimmerman G. Stuttering: a disorder of movement. J Speech Hear Res 1980;23:122-136

4. Smith A, Kelly E. Stuttering: a dynamic, multifactorial model. In Curlee RF, Siegel GM, eds. Nature and Treatment of Stuttering. Needham Heights, MA: Allyn and Bacon; 1997

5. Smith A. Stuttering: A unified approach to a multifactorial, dynamic disorder. In Bernstein Ratner N, Healey EC, eds. Stuttering Research and Practice: Bridging the Gap. Mahwah, NJ: Lawrence Erlbaum Associates, Inc; 1999:27-44

6. Individuals with Disabilities Education Act (IDEA) Amendments, 20 USC 1401(3)(A) and (B), 1401(26)

7. Riley G, Riley J. A revised component model for diagnosing and treating children who stutter. Contemp Issues Commun Sci Disord 2000;27: 188-199

8. Conture EG. Stuttering: Its Nature, Diagnosis, and Treatment. Needham Heights, MA: Allyn \& Bacon; 2001 
9. Riley G. Stuttering Severity Instrument of Children and Adults-Third Edition (SSI-3). Austin TX: Pro-Ed; 1994

10. Lewis KE. Do SSI-3 scores adequately reflect observations of stuttering behaviors? Am J SpeechLanguage Pathol 1995;4:46-59

11. Conture E. Working effectively with school children (K-12) who stutter. Short course presented at the American Speech-Language-Hearing Association; Nov. 15-18, 2001; New Orleans, LA

12. Burks H. Burks Behavior Rating Scales. Los Angeles, CA: Western Psychological Services; 1976

13. De Nil LF, Brutten GJ. Speech-associated attitudes of stuttering and nonstuttering children. J Speech Hear Res 1991;34:60-66

14. McDevitt S, Carey W. Behavioral Style Questionnaire. West Chester, PA: Temperametrics; 1995
15. Brutten E, Shoemaker D. Speech Situation Checklist. In The Southern Illinois Behavior Checklist. Carbondale, IL: Southern Illinois University; 1974

16. Andre S, Guitar G. The A-19 scale for children who stutter. In Peters T, Guitar B. Stuttering, An Integrated Approach to Its Nature and Treatment. Baltimore, MD: Williams \& Wilkins; 1991:179

17. Brutten E, Shoemaker D. Fear Survey Schedule. In The Southern Illinois Behavior Checklist. Carbondale, IL: Southern Illinois University; 1974

18. Cooper E. Personalized Fluency Control Therapy. Allen, TX: DLM Teaching Resources; 1985

19. Goldberg SA. Behavioral Cognitive Therapy. Tigard, OR: CC Publications; 1981 
